\title{
Alertness and cognitive control: Interactions in the spatial Stroop task
}

\author{
Darryl W. Schneider ${ }^{1}$
}

Published online: 19 February 2020

(C) The Psychonomic Society, Inc. 2020

\begin{abstract}
Cognitive control over information processing can be implemented by selective attention, but it is often suboptimal, as indicated by congruency effects arising from processing of irrelevant stimulus features. Research has revealed that congruency effects in some tasks are larger when subjects are more alert, and it has been suggested that this alerting-congruency interaction might be associated with spatial information processing. The author investigated the generality of the interaction by conducting a preregistered set of four experiments in which alertness was manipulated in variants of the spatial Stroop task, which involved classifying the spatial meaning of a stimulus presented at an irrelevant position. Regardless of stimulus type (arrows or words) and spatial dimension (horizontal or vertical), significant alerting-congruency interactions for response times were found in all experiments. The results are consistent with the suggestion that spatial attention and spatial information processing are important sources of the interaction, with implications for understanding how alertness is related to cognitive control.
\end{abstract}

Keywords Alertness $\cdot$ Cognitive control $\cdot$ Selective attention $\cdot$ Spatial information processing $\cdot$ Spatial Stroop task

Selective attention is a key mechanism for cognitive control. By focusing attention on relevant stimulus features and ignoring irrelevant features, the cognitive system has some control over what information is processed while pursuing a task goal. However, limitations on control are evident when task performance is influenced by irrelevant information. In various cognitive control tasks that involve selective attention, such as the flanker task (Eriksen \& Eriksen, 1974) and the Stroop task (Stroop, 1935), performance is worse when relevant and irrelevant stimulus features are associated with different responses (incongruent) than with the same response (congruent). These congruency effects indicate that irrelevant features are often attended and processed to some extent, reflecting suboptimal cognitive control. By studying congruency effects and how they are modulated by different experimental variables, researchers have sought a better understanding of control via selective attention. In the present study, I investigate the generality of the modulation of congruency effects by alertness, which is important for understanding the relationship between alertness and cognitive control - two major components of the human attention system (Posner \& Boies, 1971; Posner \& Petersen, 1990).

Darryl W. Schneider

dws@purdue.edu

1 Department of Psychological Sciences, Purdue University, 703 Third Street, West Lafayette, IN 47907, USA
This research is motivated by an unusual finding that originated with variants of the Attention Network Test (ANT; Fan, McCandliss, Sommer, Raz, \& Posner, 2002). At its core, the ANT involves an arrow flanker task: Subjects make a spatially compatible response to classify the direction of a target arrow presented among distractor arrows. The target and distractor arrows point in different directions (incongruent) or in the same direction (congruent). The frequent finding of congruency effects indicates that subjects are unable to focus attention solely on the target, resulting in distractor processing that influences task performance. Different types of cues can be presented to manipulate alertness or orienting of attention in the ANT (Fan et al., 2002). Of particular relevance in the present context, alertness is manipulated by briefly presenting alerting cues (e.g., visual signals at the potential positions of the target arrow) shortly before the arrow stimuli. ${ }^{1}$ Response times (RTs) are usually shorter on these alert trials than on noalert trials, suggesting that the cues temporarily boosted alertness (Posner, 2008). Curiously, this overarching benefit of

\footnotetext{
${ }^{1}$ Auditory signals (e.g., tones) have also been used as alerting cues in variants of the ANT and in related paradigms (e.g., Callejas, Lupiáñez, \& Tudela, 2004; Fischer, Plessow, \& Kiesel, 2010, 2012; Seibold, 2018; Weinbach \& Henik, 2012). However, as noted by Schneider (2019a), the combination of auditory alerting cues and visual stimuli confounds the alerting manipulation with modality switching (i.e., attention switches between auditory and visual modalities during alert trials, but not during no-alert trials). Modality switching is known to affect task performance (e.g., Lukas, Philipp, \& Koch, 2010; Quinlan \& Hill, 1999), suggesting that the modality of the alerting cues might be important to consider in decisions about experiment design.
} 
heightened alertness comes with a cost: Congruency effects are often larger on alert trials than on no-alert trials in variants of the ANT (e.g., Callejas, Lupiáñez, Funes, \& Tudela, 2005; Fan et al., 2002; J. W. MacLeod et al., 2010; McConnell \& Shore, 2011; Redick \& Engle, 2006; Schneider, 2018a, 2018b, 2019b; Weinbach \& Henik, 2012). This alerting-congruency interaction, which is the focus of the present study, suggests that increased alertness is associated with impaired selective attention. More generally, it indicates that different components of attention do not seem to operate independently in some situations, making it important to ascertain how they function together as parts of the attention system.

The alerting-congruency interaction implies that alertness is connected to cognitive control, but the nature of the connection is unclear. Several hypotheses have been proposed for the interaction (for an overview, see Seibold, 2018), some of which I discuss later, but none can account for all the available data (see Schneider, 2019b). A major challenge is to explain why the interaction occurs in some cognitive control tasks, but not in others. As noted earlier, the alerting-congruency interaction is routinely found in the arrow flanker task at the core of the ANT. ${ }^{2}$ Three other cognitive control tasks in which alertness has been manipulated are the Simon, global/local, and color Stroop tasks.

The Simon task (Simon \& Small, 1969; for a review, see Lu $\&$ Proctor, 1995) involves making a spatial response to classify a feature (e.g., color) of a stimulus that appears at a lateralized position (e.g., a blue square presented left of fixation). Stimulus position is irrelevant, but performance is worse when it mismatches the side of the response (incongruent) than when it matches (congruent). This congruency effect indicates that irrelevant position information is processed and influences task performance. I am aware of four experiments in which alertness was manipulated in the Simon task, and alerting-congruency interactions were found in all of them (Böckler, Alpay, \& Stürmer, 2011; Fischer et al., 2010; R. M. Klein \& Ivanoff, 2011; Soutschek, Müller, \& Schubert, 2013). These results indicate that the interaction generalizes beyond the arrow flanker task.

The global/local task (Navon, 1977) involves making a manual response to classify either the global form of a stimulus or its local elements. For example, if the stimulus is a large arrow composed of multiple small arrows, then the global task is to classify the direction of the large arrow, and the local task is to classify the direction of the small arrows. For each task, the other dimension is irrelevant, but performance is worse when it mismatches the relevant dimension (incongruent) than when it matches (congruent). This congruency effect indicates that irrelevant dimension information (which is spatial

\footnotetext{
${ }^{2}$ As discussed later, it has not yet been clearly established whether the alerting-congruency interaction regularly occurs in flanker tasks with nonarrow stimuli.
}

information for arrow stimuli) is processed and influences task performance. Weinbach and Henik (2014) manipulated alertness in an experiment with an arrow version of the global/ local task, and typical alerting-congruency interactions were found when the irrelevant dimension was salient (see also Weinbach \& Henik, 2011), whereas reversed interactions were found when the relevant dimension was salient. These results indicate that the interaction generalizes in a conditional way to the global/local task.

The color Stroop task (Stroop, 1935; for a review, see C. M. MacLeod, 1991) involves making a vocal or manual response to classify the ink color of a color word (e.g., the word GREEN printed in red ink). Word meaning is irrelevant, but performance is worse when it mismatches the ink color (incongruent) than when it matches (congruent). This congruency effect indicates that irrelevant semantic information is processed and influences task performance. Schneider (2019a) manipulated alertness in eight experiments using variants of the color Stroop task, and the typical alertingcongruency interaction was not found in any of them, replicating and extending the findings of two previous experiments (Soutschek et al., 2013; Weinbach \& Henik, 2012). These results indicate a potential constraint on the generality of the interaction.

Why does the alerting-congruency interaction occur in the arrow flanker task, the Simon task, and conditionally in the global/local task, but not in the color Stroop task? Recently, I suggested that there is "a spatial attention constraint on the relationship between alertness and cognitive control, part of which might involve having a task goal associated with spatial information processing" (Schneider, 2019a, p. 119). A distinction can be made between spatial information processing associated with stimuli and responses. Depending on the stimuli, perceptual classification involves spatial information processing (e.g., discriminating between line orientations) or it does not (e.g., discriminating between colors). Depending on the responses, selection of a motor program involves spatial information processing (e.g., choosing between left and right key-press responses) or it does not (e.g., choosing between vocal responses). I suggest that the alerting-congruency interaction might arise when spatial information processing is needed to accurately classify the relevant stimulus feature, prior to mapping that classification to a response.

From this perspective, it is potentially fruitful to consider the involvement of spatial attention and spatial information processing in the aforementioned cognitive control tasks. Spatial attention is needed in the arrow flanker task because the target is spatially separated from the distractors. Spatial information processing occurs because the task is to classify the direction of the target arrow. Spatial attention is needed in the Simon task because attention has to shift to the lateralized position of the stimulus in order to classify the relevant stimulus feature. Whether spatial information processing occurs 
for classification of the relevant feature in the Simon task depends on the stimuli. ${ }^{3}$ It is unlikely that spatial information is needed for classification of colors (e.g., red vs. green lights; Craft \& Simon, 1970). However, some spatial information processing occurs when classifying shapes. For example, in two of the experiments in which alertness was manipulated in the Simon task (Böckler et al., 2011; Fischer et al., 2010, Experiment 2), subjects classified the shape of the stimulus, which was a square or a diamond. Shape perception involves processing multiple spatial properties of stimuli (Palmer, 1999), and the only difference between a square and a diamond is a spatial transformation (rotation). Thus, even though shape classification (square vs. diamond) does not yield position information, it does involve spatial information processing. Spatial attention is needed in the global/local task because attention has to focus on either the global form of the stimulus or its local elements. Spatial information processing occurs in the arrow versions of the global/local task studied by Weinbach and Henik $(2011,2014)$ because the task is to classify the direction of either the large, global arrow or the small, local arrows.

In contrast, spatial attention and spatial information processing play little or no role in the color Stroop task. Subjects have to attend to the stimulus display to perform the task, but spatial attention does not have to be shifted (if the stimulus is always presented centrally) and it cannot be used to distinguish between relevant and irrelevant features (if they are spatially integrated; e.g., the word GREEN printed in red ink). Schneider (2019a) did not obtain typical alertingcongruency interactions in the color Stroop task even when the relevant and irrelevant features were spatially separated (e.g., a red rectangle flanked above and below by the word $G R E E N)$. Spatial information is not needed for classifying the relevant feature in the color Stroop task. Moreover, Schneider (2019a) did not obtain typical alerting-congruency interactions in color Stroop-like tasks in which the irrelevant feature conveyed spatial information (e.g., a red rectangle flanked above and below by the word $L E F T$ ), ruling out the notion that irrelevant spatial information processing is sufficient for producing the alerting-congruency interaction.

The preceding analysis seems consistent with the idea that spatial information processing of the relevant stimulus feature is an important determinant of whether the alertingcongruency interaction is obtained in a specific cognitive control task. Spatial information processing of the relevant feature definitely occurs in the arrow flanker task and in arrow versions of the global/local task, whereas it occurs to varying degrees in the Simon task. Alerting-congruency interactions have been obtained with all of those tasks. Spatial information

\footnotetext{
${ }^{3}$ Regardless of the relevant feature to be classified, spatial information processing must occur for the irrelevant feature (stimulus position); otherwise, there would be no congruency effect at all in the Simon task.
}

processing of the relevant feature arguably does not occur in the color Stroop task, and alerting-congruency interactions have not been obtained with that task. However, if the Stroop task were modified such that spatial information was needed to achieve the task goal, then alerting-congruency interactions should emerge. This possibility can be explored by manipulating alertness in the spatial Stroop task.

There are many variants of the spatial Stroop task, which differs from the Simon task by having both the relevant and irrelevant stimulus features convey spatial information ( $\mathrm{Lu} \&$ Proctor, 1995). For example, Logan and Zbrodoff (1979) studied a spatial Stroop task in which subjects classified the meaning of a word (ABOVE or BELOW) that was positioned above or below fixation. Stimulus position was irrelevant, but performance was worse when it mismatched the word's meaning (incongruent) than when it matched (congruent). Similar results have been obtained with classification of nonlinguistic stimuli, such as arrows (e.g., Clark \& Brownell, 1975). The congruency effect in the spatial Stroop task is similar to that of the Simon task, in that irrelevant position information is the source of the effect. However, the spatial Stroop task involves the explicit goal of classifying spatial information conveyed by the relevant stimulus feature, whereas the Simon task does not. The congruency effect in the spatial Stroop task is analogous to that of the color Stroop task, except that the irrelevant semantic information influencing task performance is spatial rather than color information.

I am aware of only one experiment in which alertness was manipulated in the spatial Stroop task. Fischer et al. (2010, Experiment 1) studied a spatial Stroop task in which 26 subjects classified the direction of a left-pointing or right-pointing arrow that was positioned to the left or right of a central fixation sign. ${ }^{4}$ The arrow's direction and position either matched (congruent) or mismatched (incongruent). Trials were divided into prime-probe pairs, with an alerting cue (a tone) presented before the arrow on half of the prime trials. A main finding was that congruency effects on prime trials were larger with alerting cues than without them. This result represents initial evidence that the alerting-congruency interaction generalizes to the spatial Stroop task. However, it is unknown whether Fischer et al.'s finding can be replicated with a more typical experiment design that involves visual alerting cues and no prime-probe pairing of trials.

For the present study, I conducted a preregistered, multiexperiment investigation of alerting effects in variants of the spatial Stroop task. ${ }^{5}$ In each experiment, subjects made

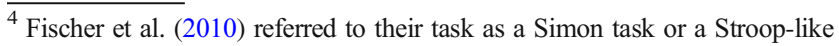
Simon task, but it is most consistent with Lu and Proctor's (1995) definition of a spatial Stroop task.

${ }^{5}$ All experiments were preregistered and the protocol is publicly available (https://aspredicted.org/nn2iz.pdf). The protocol describes the present study's purpose, experiment design (including the differences between experiments), independent and dependent variables, sample size, data exclusion criteria, and planned statistical analyses. The protocol was not peer reviewed, but it was registered before data collection commenced.
} 
a spatially compatible key-press response to classify the spatial meaning of a stimulus presented at an irrelevant position. Stimulus meaning and position either matched (congruent) or mismatched (incongruent) on each trial. Stimulus type (arrows or words) and spatial dimension (horizontal or vertical) were varied between experiments. Figure 1 shows examples of incongruent stimulus displays for all experiments, as well as the responses for each spatial dimension. I manipulated stimulus type and spatial dimension to assess the replicability and generality of the results across different task conditions, which is important in the context of concerns about the replicability of findings in psychology (e.g., R. A. Klein et al., 2018; Open Science Collaboration, 2015).

In Experiment 1 (arrow stimuli; horizontal), subjects pressed a left or right key to classify the direction of a leftpointing or right-pointing arrow, positioned left or right of fixation (e.g., top-left display of Fig. 1), similar to the task in Fischer et al. (2010). In Experiment 2 (arrow stimuli; vertical), subjects pressed an up or down key to classify the direction of an up-pointing or down-pointing arrow, positioned above or below fixation (e.g., top-right display of Fig. 1). In Experiment 3 (word stimuli; horizontal), subjects pressed a left or right key to classify the meaning of the word $L E F T$ or RIGHT, positioned left or right of fixation (e.g., middle-left display of Fig. 1). In Experiment 4 (word stimuli; vertical), subjects pressed an up or down key to classify the meaning of the word $U P$ or $D O W N$, positioned above or below fixation (e.g., middle-right display of Fig. 1).

Besides congruency, each experiment also included an alerting manipulation. On no-alert trials, the stimulus was presented after a variable fixation interval, which is a standard feature of the ANT (Fan et al., 2002) and several previous studies on the alerting-congruency interaction (e.g., Fischer et al., 2010; Schneider, 2018a, 2018b, 2019a, 2019b; Seibold, 2018; Soutschek et al., 2013). On alert trials (see Fig. 2), visual alerting cues (squares) were briefly presented at the two potential stimulus positions, $500 \mathrm{~ms}$ before stimulus onset. These alerting cues are similar to the double cues used to manipulate alertness in the ANT (Fan et al., 2002), and the 500 -ms alerting interval is a standard feature of the ANT and my previous studies on the alerting-congruency interaction (Schneider, 2018a, 2018b, 2019a, 2019b).

The alerting manipulation enabled me to assess the presence or absence of an alerting-congruency interaction in each

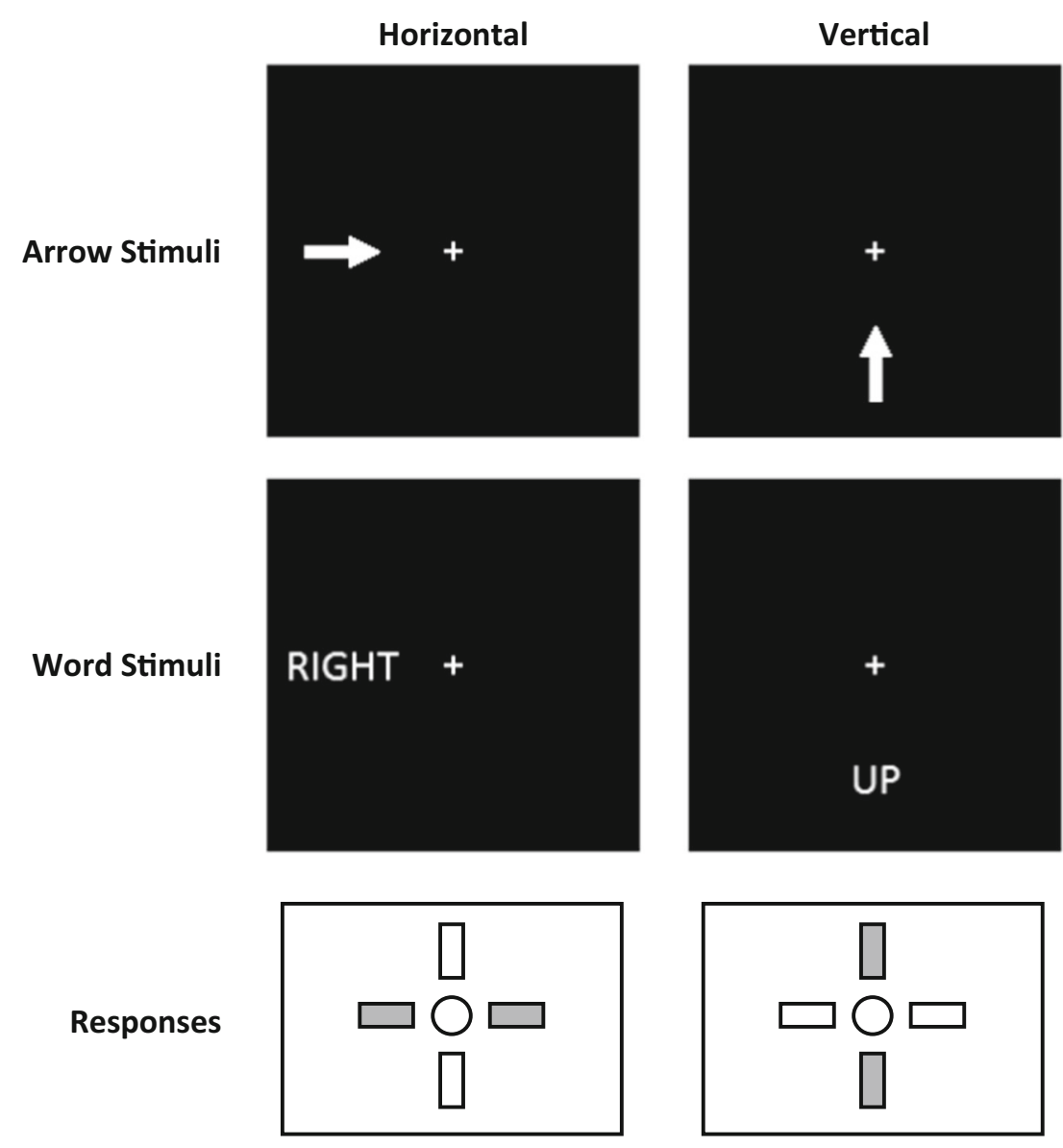

Fig. 1 Top and middle rows: Example stimulus displays (drawn to scale) as a function of stimulus type (arrows or words) and spatial dimension (horizontal or vertical). All examples depict incongruent stimuli. Bottom

row: Designated response keys (shaded in gray) on schematic displays of the response pad as a function of spatial dimension 


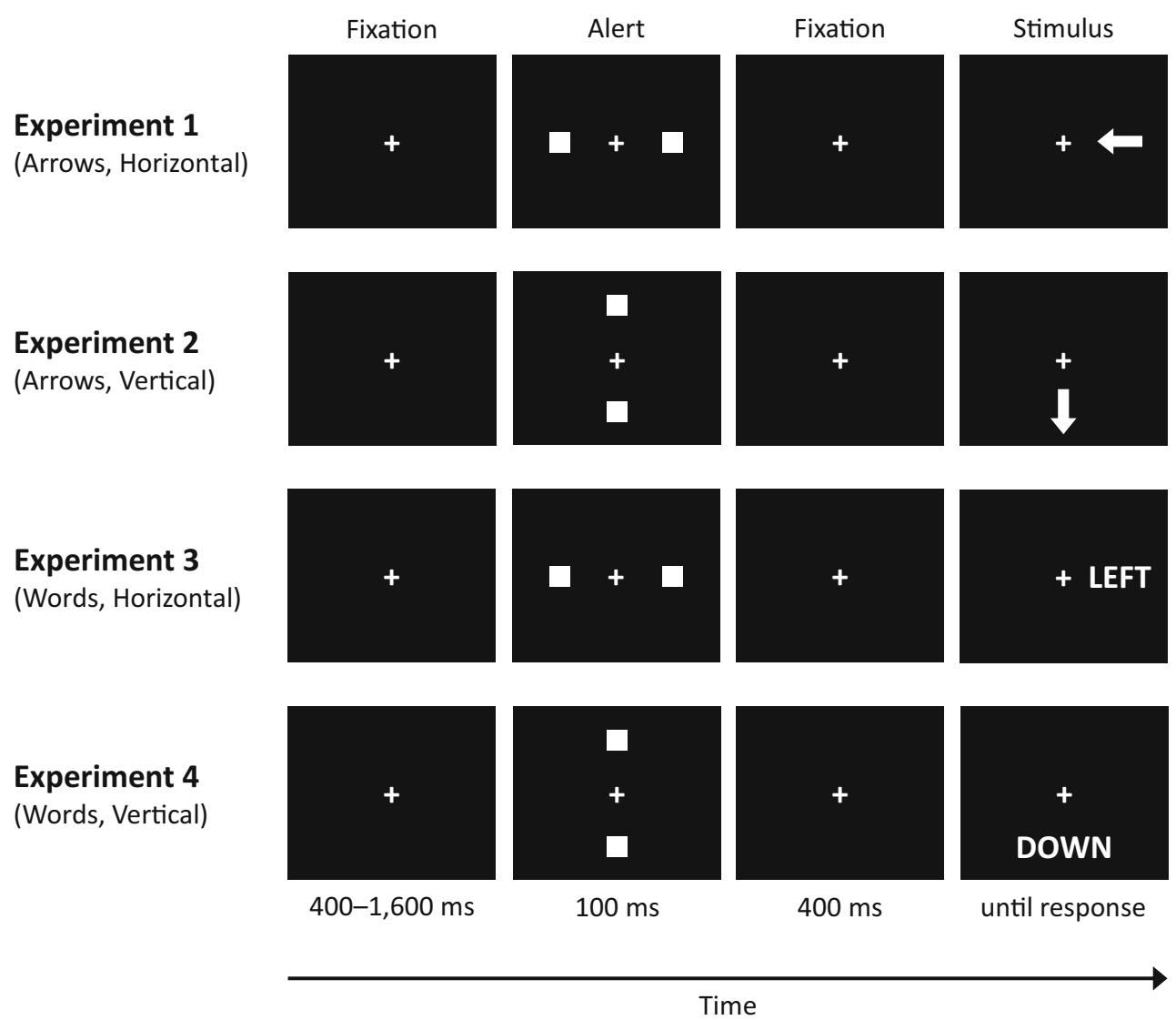

Fig. 2 Example sequences of displays (not drawn to scale) on alert trials for each experiment. The sequences were identical on no-alert trials, except that only the central fixation cross appeared in the second display (i.e., the square alerting cues were absent)

experiment. Based on my earlier analysis, if spatial information processing of the relevant stimulus feature is an important determinant of whether the interaction is obtained in a cognitive control task, then the interaction should be present in all variants of the spatial Stroop task investigated here. In each case, spatial information processing is needed to achieve the task goal, regardless of the spatial dimension (horizontal or vertical) and whether the relevant spatial information is conveyed by linguistic or nonlinguistic stimuli (words or arrows, respectively). The reliable occurrence of the alertingcongruency interaction in the spatial Stroop task would help to clarify its generality across tasks and inform theorizing about the relationship between alertness and cognitive control.

\section{Method}

\section{Subjects}

A total of 240 undergraduate students from Purdue University participated for course credit. There were 60 subjects per experiment (Experiment 1: 36 female, 53 right-handed, mean age $=19.3$ years; Experiment 2: 30 female, 51 right-handed, mean age $=19.2$ years; Experiment 3: 31 female, 54 right- handed, mean age $=19.6$ years; Experiment 4: 33 female, 52 right-handed, mean age $=19.3$ years). The preregistered sample size for each experiment was based on a power analysis (calculated using G*Power 3.1; Faul, Erdfelder, Lang, \& Buchner, 2007) that indicated 59 subjects would provide $90 \%$ power to detect an alerting-congruency interaction with an effect size of $d=0.43$, which is the overall effect size reported by Schneider (2019a) from a meta-analysis of nine experiments involving arrow flanker tasks. The sample size was rounded up to 60 . Data were excluded from one additional subject (in Experiment 1) based on a preregistered exclusion criterion (mean RT exceeded four standard deviations of the group mean). All subjects reported having normal or corrected-to-normal vision.

\section{Apparatus}

The experiments were conducted using E-Prime 3 (Psychology Software Tools, Inc.) on computers that displayed stimuli on monitors at a viewing distance of approximately $50 \mathrm{~cm}$. Responses were registered from Cedrus RB540 response pads (Cedrus Corp.), which have five keys in a plus-sign spatial configuration (see bottom displays in Fig. 1; the circular central key was not used in any experiment). 


\section{Task, stimuli, and responses}

Each experiment used a variant of the spatial Stroop task, which involved making a spatially compatible key-press response to classify the spatial meaning of a stimulus while ignoring its position. Congruency was defined by the match (congruent) or mismatch (incongruent) between the spatial meaning of the stimulus and its position. The set of spatial Stroop tasks used in the experiments reflected a factorial manipulation of stimulus type (arrows or words) and spatial dimension (horizontal or vertical). Figure 1 shows example stimulus displays as a function of those variables, as well as the designated response keys for each spatial dimension (left and right keys for the horizontal dimension are shaded gray in the bottom-left display; up and down keys for the vertical dimension are shaded gray in the bottom-right display). Stimuli were displayed in white on a black background. Word stimuli were presented in 18-point Calibri font.

Experiment 1 The stimuli were arrows and the spatial dimension was horizontal (left-right). Subjects pressed a left or right key (see Fig. 1, bottom-left display) to classify the direction of a left-pointing or right-pointing arrow $\left(1.15^{\circ} \times 0.46^{\circ}\right)$, respectively, positioned $1.72^{\circ}$ (center-to-center distance) to the left or right of a central fixation cross $\left(0.23^{\circ} \times 0.23^{\circ}\right)$. A congruent stimulus was an arrow that pointed in a direction that matched its position relative to fixation (a left-pointing arrow positioned left of fixation, or a right-pointing arrow positioned right of fixation). An incongruent stimulus was an arrow that pointed in a direction that mismatched its position relative to fixation (a left-pointing arrow positioned right of fixation, or a right-pointing arrow positioned left of fixation; the latter is shown in Fig. 1, top-left display).

Experiment 2 The stimuli were arrows and the spatial dimension was vertical (up-down). Subjects pressed an up or down $\mathrm{key}^{6}$ (see Fig. 1, bottom-right display) to classify the direction of an up-pointing or down-pointing arrow $\left(0.46^{\circ} \times 1.15^{\circ}\right)$, respectively, positioned $1.72^{\circ}$ above or below a central fixation cross. A congruent stimulus was an arrow that pointed in a direction that matched its position relative to fixation (a down-pointing arrow positioned below fixation, or an uppointing arrow positioned above fixation). An incongruent

\footnotetext{
${ }^{6}$ The response pad was laid flat on the tabletop in front of subjects, which meant that the vertical dimension represented variation in the transverse plane for responses and the frontal plane for stimuli. However, the far and near keys on the response pad were referred to as "up" and "down" keys, respectively, in the instructions to subjects. No subjects expressed difficulty with this response coding, likely because of their familiarity with it in other contexts (e.g., "up" and "down" arrow keys on computer keyboards). Moreover, research on the vertical Simon task - a relative of the spatial Stroop task - has shown similar results for different response-device orientations (Zhong, Xiong, Vu, \& Proctor, 2018), suggesting that responses in the transverse plane are readily coded as up and down (or top and bottom) when interacting with twodimensional stimulus displays.
}

stimulus was an arrow that pointed in a direction that mismatched its position relative to fixation (a down-pointing arrow positioned above fixation, or an up-pointing arrow positioned below fixation; the latter is shown in Fig. 1, top-right display).

Experiment 3 The stimuli were words and the spatial dimension was horizontal (left-right). Subjects pressed a left or right key (see Fig. 1, bottom-left display) to classify the meaning of the word LEFT $\left(1.15^{\circ} \times 0.40^{\circ}\right)$ or RIGHT $\left(1.66^{\circ} \times 0.40^{\circ}\right)$, respectively, positioned $1.72^{\circ}$ to the left or right of a central fixation cross. A congruent stimulus was a word with a meaning that matched its position relative to fixation (LEFT positioned left of fixation, or RIGHT positioned right of fixation). An incongruent stimulus was a word with a meaning that mismatched its position relative to fixation (LEFT positioned right of fixation, or RIGHT positioned left of fixation; the latter is shown in Fig. 1, middle-left display).

Experiment 4 The stimuli were words and the spatial dimension was vertical (up-down). Subjects pressed an up or down key (see Fig. 1, bottom-right display) to classify the meaning of the word $U P\left(0.67^{\circ} \times 0.40^{\circ}\right)$ or $D O W N\left(1.72^{\circ} \times 0.40^{\circ}\right)$, respectively, positioned $1.72^{\circ}$ above or below a central fixation cross. A congruent stimulus was a word with a meaning that matched its position relative to fixation (DOWN positioned below fixation, or UP positioned above fixation). An incongruent stimulus was a word with a meaning that mismatched its position relative to fixation (DOWN positioned above fixation, or $U P$ positioned below fixation; the latter is shown in Fig. 1, middle-right display).

\section{Procedure}

Subjects were tested individually in private rooms after giving written informed consent for a study protocol approved by the Purdue University Institutional Review Board. Instructions were presented on-screen and read aloud by the experimenter. Subjects were instructed to classify the direction of the arrow (Experiments 1 and 2) or the meaning of the word (Experiments 3 and 4) on each trial, ignoring stimulus position. They were instructed to respond quickly and accurately by using their left and right index fingers to press the designated response keys, which were labeled on a photo of the response pad that appeared on-screen below the instruction text (the photo was not present after the instructions). ${ }^{7}$

\footnotetext{
${ }^{7}$ For Experiments 1 and 3, in which the spatial dimension was horizontal, the photo of the response pad included the text labels "LEFT key" and "RIGHT key" linked by lines to the left and right keys, respectively. The left and right keys are the gray-shaded keys in the schematic of the response pad shown in Fig. 1, bottom-left display. For Experiments 2 and 4, in which the spatial dimension was vertical, the photo of the response pad included the text labels "UP key" and "DOWN key" linked by lines to the up and down keys, respectively. The up and down keys are the gray-shaded keys in the schematic of the response pad shown in Fig. 1, bottom-right display.
} 
During the instructions, subjects completed eight example noalert trials (two trials for each possible stimulus display) with accuracy feedback. Afterward, they completed 12 blocks of 32 trials per block without accuracy feedback.

Alert and no-alert trials occurred equally often in each block and involved similar sequences of events. Figure 2 shows example sequences of displays on alert trials for each experiment. The first display consisted of a central fixation cross presented for a randomly selected interval of $400 \mathrm{~ms}$; $800 \mathrm{~ms} ; 1,200 \mathrm{~ms}$; or 1,600 ms. The second display consisted of the central fixation cross and two alerting cues (white-filled squares, each $0.44^{\circ} \times 0.44^{\circ}$ ) presented at the two potential stimulus positions for $100 \mathrm{~ms}$. In Experiments 1 and 3, which involved the horizontal dimension, the alerting cues appeared to the left and right of the central fixation cross. In Experiments 2 and 4, which involved the vertical dimension, the alerting cues appeared above and below the central fixation cross. The third display consisted of the central fixation cross by itself for $400 \mathrm{~ms}$. The fourth display consisted of the central fixation cross and the stimulus at a selected position, which remained on-screen until a response key was pressed. Afterward, the screen was cleared, and the first display for the next trial started immediately.

The sequences of displays on no-alert trials were identical to those shown in Fig. 2, except that the second display consisted of the central fixation cross by itself for $100 \mathrm{~ms}$ (i.e., the square alerting cues were absent). From the subject's perspective, there appeared to be a single, uninterrupted fixation display until stimulus onset on no-alert trials.

The fixation and alerting intervals were identical to those used in Schneider (2019b). The fixation intervals for the first display were randomized independently of the other variables and occurred equally often in random order within every block. All possible combinations of alerting and stimulus conditions occurred twice in random order within each consecutive subset of 16 trials.

\section{Results}

Data trimming and analyses followed the preregistered protocol. The first block of trials was excluded as practice for each experiment. Trials with RTs exceeding three standard deviations of the mean in each condition for a given subject were excluded $(<1.7 \%$ of trials in each experiment). Error trials were excluded from the RT analyses. Mean RTs and mean error rates appear as a function of alerting (no alert or alert) and congruency (incongruent or congruent) for each experiment in Table 1. The results of repeated-measures analyses of variance (ANOVAs) with those variables as factors are summarized in Table 2. All effects were significant $(\alpha=.05)$ in the RT data. Fewer effects were significant in the error data because mean
Table 1 Mean response times and mean error rates

\begin{tabular}{lllll}
\hline Experiment & Alerting & Congruency & $\begin{array}{l}\text { Response time } \\
(\mathrm{ms})\end{array}$ & Error rate (\%) \\
\hline 1 & \multirow{2}{*}{ No alert } & Incongruent & $522(11)$ & $4.3(.5)$ \\
& & Congruent & $474(10)$ & $.7(.2)$ \\
& \multirow{2}{*}{ Alert } & Incongruent & $494(10)$ & $4.1(.6)$ \\
& & Congruent & $438(8)$ & $.8(.2)$ \\
& \multirow{4}{*}{ No alert } & Incongruent & $551(10)$ & $4.0(.5)$ \\
& & Congruent & $488(8)$ & $.6(.1)$ \\
& \multirow{4}{*}{ Alert } & Incongruent & $524(10)$ & $3.1(.4)$ \\
& & Congruent & $453(8)$ & $.5(.1)$ \\
& \multirow{2}{*}{ No alert } & Incongruent & $518(9)$ & $4.1(.6)$ \\
& & Congruent & $493(9)$ & $1.5(.2)$ \\
& \multirow{2}{*}{ Alert } & Incongruent & $509(9)$ & $4.0(.6)$ \\
& & Congruent & $464(8)$ & $.9(.2)$ \\
4 & \multirow{2}{*}{ No alert } & Incongruent & $530(7)$ & $3.8(.5)$ \\
& & Congruent & $484(7)$ & $.9(.1)$ \\
& \multirow{2}{*}{ Alert } & Incongruent & $512(7)$ & $3.8(.5)$ \\
& & Congruent & $451(6)$ & $.6(.1)$ \\
\hline
\end{tabular}

Note. Standard errors of the means appear in parentheses

error rates were generally low $(<2.7 \%$ in each experiment). With two exceptions (noted later), the error data patterns were consistent with the RT data patterns; therefore, I focus on the latter. Mean RTs are presented visually in Fig. 3.

The ANOVA results are supplemented by Bayes-factor analyses of the interactions between alerting and congruency (scaled JZS Bayes factors with $r=1$; Rouder, Speckman, Sun, Morey, \& Iverson, 2009). Each Bayes factor (BF) quantifies the strength of evidence for the presence or absence of an alerting-congruency interaction. Following the notation and categorization used by Schneider (2019a, 2019b), $B F_{\text {alt }}$ indicates the data are more likely under the alternative hypothesis that an interaction is present, whereas $B F_{\text {null }}$ indicates the data are more likely under the null hypothesis that an interaction is absent. ${ }^{8}$ The $B F$ subscript is determined by the data, but one can convert from $B F_{\text {alt }}$ to $B F_{\text {null, }}$, or vice versa, by inverting the $B F$ value (e.g., $B F_{\text {null }}=1 / B F_{\text {alt }}$ ). The $B F$ value indicates how many times more likely the data are under one hypothesis than the other, which can be categorized as weak (1-3), moderate (3-10), strong (10-30), very strong (30-100), or decisive ( $>100$ ) evidence. By expressing $B F$ values in terms of the hypothesis (null or alternative) under which the data are more likely, one avoids fractional values that might be difficult to interpret (Siegler \& Lortie-Forgues, 2017).

\footnotetext{
${ }^{8} B F_{\text {alt }}$ and $B F_{\text {null }}$ correspond to $B F_{10}$ and $B F_{01}$, respectively, in other studies (e.g., Schönbrodt \& Wagenmakers, 2018).
} 
Table 2 Summary of individual experiment analyses of variance

\begin{tabular}{|c|c|c|c|c|c|c|c|}
\hline \multirow[t]{2}{*}{ Experiment } & \multirow[t]{2}{*}{ Effect } & \multicolumn{3}{|c|}{ Response time } & \multicolumn{3}{|l|}{ Error rate } \\
\hline & & $F(1,59)$ & $p$ & $\eta_{\mathrm{p}}^{2}$ & $F(1,59)$ & $p$ & $\eta_{p}^{2}$ \\
\hline \multirow[t]{3}{*}{1} & $\mathrm{~A}$ & 94.54 & $<.001$ & .616 & .07 & .789 & .001 \\
\hline & $\mathrm{C}$ & 128.33 & $<.001$ & .685 & 71.37 & $<.001$ & .547 \\
\hline & $\mathrm{A} \times \mathrm{C}$ & 4.58 & .037 & .072 & .48 & .493 & .008 \\
\hline \multirow[t]{3}{*}{2} & $\mathrm{~A}$ & 135.92 & $<.001$ & .697 & 5.10 & .028 & .080 \\
\hline & $\mathrm{C}$ & 346.94 & $<.001$ & .855 & 60.02 & $<.001$ & .504 \\
\hline & $\mathrm{A} \times \mathrm{C}$ & 5.21 & .026 & .081 & 4.56 & .037 & .072 \\
\hline \multirow[t]{3}{*}{3} & A & 85.44 & $<.001$ & .592 & 2.85 & .097 & .046 \\
\hline & $\mathrm{C}$ & 145.94 & $<.001$ & .712 & 39.01 & $<.001$ & .398 \\
\hline & $\mathrm{A} \times \mathrm{C}$ & 23.72 & $<.001$ & .287 & 1.41 & .240 & .023 \\
\hline \multirow[t]{3}{*}{4} & $\mathrm{~A}$ & 108.12 & $<.001$ & .647 & 1.01 & .318 & .017 \\
\hline & $\mathrm{C}$ & 448.63 & $<.001$ & .884 & 58.56 & $<.001$ & .498 \\
\hline & $\mathrm{A} \times \mathrm{C}$ & 25.62 & $<.001$ & .303 & .65 & .425 & .011 \\
\hline
\end{tabular}

Note. $\mathrm{A}=$ Alerting; $\mathrm{C}=$ Congruency

\section{Individual experiment analyses}

Experiment 1 (arrow stimuli; horizontal) Subjects were faster on alert trials (mean RT of $466 \mathrm{~ms}$ ) than on no-alert trials (498 $\mathrm{ms})$, and they were slower for incongruent stimuli (508 ms) than for congruent stimuli (456 ms), resulting in significant main effects of alerting and congruency. As shown in Fig. 3 (top-left panel), congruency effects were larger on alert trials (56 $\mathrm{ms}$ ) than on no-alert trials $(48 \mathrm{~ms})$, resulting in a significant interaction $\left(B F_{\text {null }}=1.1\right)$.

Experiment 2 (arrow stimuli; vertical) Subjects were faster on alert trials (489 ms) than on no-alert trials (519 ms), and they were slower for incongruent stimuli $(538 \mathrm{~ms})$ than for congruent stimuli (471 ms), resulting in significant main effects of alerting and congruency. As shown in Fig. 3 (top-right panel), congruency effects were larger on alert trials $(71 \mathrm{~ms})$ than on no-alert trials $(63 \mathrm{~ms})$, resulting in a significant interaction $\left(B F_{\text {alt }}=1.2\right)^{9}$

Experiment 3 (word stimuli; horizontal) Subjects were faster on alert trials (486 ms) than on no-alert trials (506 ms), and they were slower for incongruent stimuli $(513 \mathrm{~ms})$ than for congruent stimuli (479 ms), resulting in significant main effects of alerting and congruency. As shown in Fig. 3 (bottomleft panel), congruency effects were larger on alert trials (44 $\mathrm{ms})$ than on no-alert trials $(25 \mathrm{~ms})$, resulting in a significant interaction $\left(B F_{\text {alt }}=1,864 \cdot 2\right)$.

\footnotetext{
$\overline{9}$ There was also a significant interaction in the error data (see Tables 1 and 2), but in the opposite direction: congruency effects were smaller on alert trials $(2.6 \%)$ than on no-alert trials $(3.4 \%), B F_{\text {null }}=1.1$. This result was not replicated in any of the other experiments, suggesting that it might be spurious.
}

Experiment 4 (word stimuli; vertical) Subjects were faster on alert trials (481 ms) than on no-alert trials $(507 \mathrm{~ms})$, and they were slower for incongruent stimuli $(521 \mathrm{~ms})$ than for congruent stimuli (467 ms), resulting in significant main effects of alerting and congruency. As shown in Fig. 3 (bottom-right panel), congruency effects were larger on alert trials (61 ms) than on no-alert trials (46 ms), resulting in a significant interaction $\left(B F_{\text {alt }}=3,605.7\right)$.

\section{Combined experiment analyses}

Drawing on the factorial structure of the set of experiments, preregistered secondary analyses for all experiments combined were conducted by performing 2 (stimulus type: arrows or words) $\times 2$ (spatial dimension: horizontal or vertical) $\times 2$ (alerting: no alert or alert) $\times 2$ (congruency: incongruent or congruent) mixed ANOVAs, with the first two factors between subjects. The ANOVA results are summarized in Table 3. To avoid redundancy with the individual experiment analyses, I focus on effects involving stimulus type and spatial dimension.

Alerting effects were larger for arrow stimuli $(31 \mathrm{~ms})$ than for word stimuli (22 $\mathrm{ms})$, resulting in a significant interaction between stimulus type and alerting. As shown in Fig. 3 (top vs. bottom panels), congruency effects were larger for arrow stimuli (59 ms) than for word stimuli (44 ms), resulting in a significant interaction between stimulus type and congruency. Also shown in Fig. 3 (left vs. right panels), congruency effects were larger for the vertical dimension $(61 \mathrm{~ms})$ than for the horizontal dimension (43 ms), resulting in a significant interaction between spatial dimension and congruency. Alertingcongruency interactions (differences in congruency effects between alert and no-alert trials) were larger for word stimuli (17 $\mathrm{ms})$ than for arrow stimuli $(8 \mathrm{~ms})$, resulting in a significant three-way interaction between stimulus type, alerting, and congruency. ${ }^{10}$

\section{Discussion}

Cognitive control over information processing can be implemented by selective attention, but it is often suboptimal, as indicated by congruency effects arising from processing of irrelevant stimulus features. Research has revealed that congruency effects in some tasks are modulated by alertness, but in an unusual way: Congruency effects are often larger when subjects are more alert, despite an overall performance benefit associated with heightened alertness (e.g., Callejas et al.,

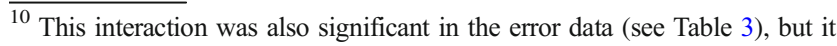
reflected a slightly different pattern: alerting-congruency interactions occurred in the typical direction for word stimuli $(0.4 \%)$ and in the reverse direction for arrow stimuli $(-0.6 \%)$, with the latter driven primarily by the Experiment 2 data (see footnote 9 ).
} 

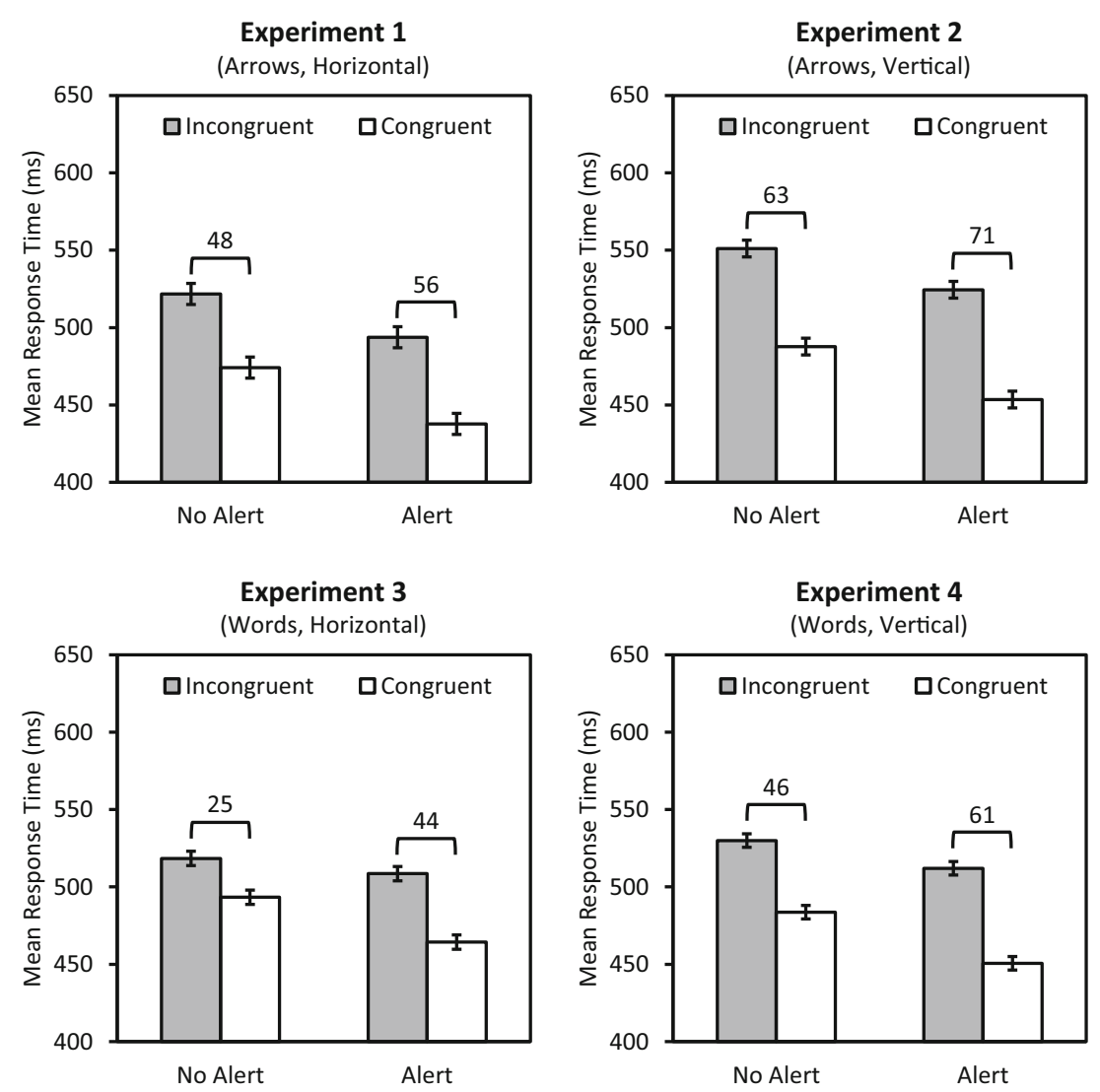

Fig. 3 Mean response times as a function of alerting (no alert or alert) and congruency (incongruent or congruent) for each experiment. Numbers above bars indicate congruency effects. Error bars represent 95\% confidence intervals (Masson \& Loftus, 2003)

Table 3 Summary of combined experiment analyses of variance

\begin{tabular}{|c|c|c|c|c|c|c|}
\hline \multirow[t]{2}{*}{ Effect } & \multicolumn{3}{|c|}{ Response time } & \multicolumn{3}{|l|}{ Error rate } \\
\hline & $F(1,236)$ & $p$ & $\eta_{\mathrm{p}}^{2}$ & $F(1,236)$ & $p$ & $\eta_{\mathrm{p}}^{2}$ \\
\hline $\mathrm{S}$ & .06 & .802 & $<.001$ & .46 & .498 & .002 \\
\hline $\mathrm{D}$ & 1.49 & .223 & .006 & 2.21 & .139 & .009 \\
\hline $\mathrm{A}$ & 410.07 & $<.001$ & .635 & 6.98 & .009 & .029 \\
\hline $\mathrm{C}$ & 885.48 & $<.001$ & .790 & 223.20 & $<.001$ & .486 \\
\hline $\mathrm{S} \times \mathrm{D}$ & 2.17 & .142 & .009 & .02 & .900 & $<.001$ \\
\hline $\mathrm{S} \times \mathrm{A}$ & 11.14 & .001 & .045 & $<.01$ & .986 & $<.001$ \\
\hline $\mathrm{S} \times \mathrm{C}$ & 19.09 & $<.001$ & .075 & .59 & .442 & .003 \\
\hline $\mathrm{D} \times \mathrm{A}$ & .65 & .420 & .003 & .35 & .554 & .001 \\
\hline $\mathrm{D} \times \mathrm{C}$ & 24.53 & $<.001$ & .094 & .11 & .742 & $<.001$ \\
\hline $\mathrm{A} \times \mathrm{C}$ & 49.61 & $<.001$ & .174 & .13 & .717 & .001 \\
\hline $\mathrm{S} \times \mathrm{D} \times \mathrm{A}$ & 2.15 & .143 & .009 & 2.20 & .139 & .009 \\
\hline $\mathrm{S} \times \mathrm{D} \times \mathrm{C}$ & .30 & .583 & .001 & .55 & .460 & .002 \\
\hline $\mathrm{S} \times \mathrm{A} \times \mathrm{C}$ & 6.53 & .011 & .027 & 5.48 & .020 & .023 \\
\hline $\mathrm{D} \times \mathrm{A} \times \mathrm{C}$ & .46 & .498 & .002 & .64 & .423 & .003 \\
\hline $\mathrm{S} \times \mathrm{D} \times \mathrm{A} \times \mathrm{C}$ & .20 & .652 & .001 & .14 & .705 & .001 \\
\hline
\end{tabular}

Note. $\mathrm{S}=$ Stimulus Type; $\mathrm{D}=$ Spatial Dimension; $\mathrm{A}=$ Alerting; $\mathrm{C}=$ Congruency
2005; Fan et al., 2002; J. W. MacLeod et al., 2010; McConnell \& Shore, 2011; Redick \& Engle, 2006; Schneider, 2018a, 2018b, 2019b; Weinbach \& Henik, 2012). The mechanism underlying this alerting-congruency interaction is unclear, in part because the interaction is obtained with some cognitive control tasks (e.g., arrow flanker, Simon, and global/local tasks), but not with others (color Stroop task). Drawing on the idea that spatial attention and spatial information processing might be important for eliciting the alerting-congruency interaction (Schneider, 2019a), the purpose of the present study was to determine whether the interaction reliably occurs in the spatial Stroop task.

I conducted a set of four experiments in which alertness was manipulated in variants of the spatial Stroop task. In each experiment, subjects classified the spatial meaning of a stimulus presented at an irrelevant position that was either congruent or incongruent with the meaning. Stimulus type (arrows or words) and spatial dimension (horizontal or vertical) varied between experiments (see Fig. 1). Alerting cues preceded the stimuli on half of the trials in each experiment (see Fig. 2). The RT data patterns were highly consistent across the four experiments (see Fig. 3). Subjects responded slower to incongruent stimuli than to congruent stimuli, replicating congruency effects found in previous studies involving the spatial Stroop task (e.g., Clark \& Brownell, 1975; Logan \& Zbrodoff, 
1979; Palef \& Olson, 1975). The congruency effects were larger when classifying arrows instead of words, and when the spatial dimension for the task was vertical instead of horizontal. Subjects responded faster on alert trials than on noalert trials, replicating alerting effects found in previous studies with visual alerting cues (e.g., Fan et al., 2002; Redick \& Engle, 2006; Schneider, 2018a).

Critically, there was a significant alerting-congruency interaction in every experiment (see Table 2). Bayes-factor analyses indicated that the evidence in favor of an interaction was weak for arrow stimuli, but decisive for word stimuli. The finding of an alerting-congruency interaction in Experiment 1 , which involved arrow stimuli in a horizontal version of the spatial Stroop task, replicates the results of Fischer et al. (2010), but with visual alerting cues and more than double their sample size. The alerting-congruency interactions found in Experiments 2-4 go beyond Fischer et al.'s results by providing novel evidence that the interaction generalizes to word stimuli and to vertical versions of the spatial Stroop task. To my knowledge, the present study is the first preregistered, multiexperiment demonstration of reliable alertingcongruency interactions in a cognitive control task other than the arrow flanker task.

\section{Comparing Stroop tasks and stimuli}

The present results fit with the idea that spatial attention and spatial information processing are important sources of the alerting-congruency interaction (Schneider, 2019a). Spatial attention is needed in the spatial Stroop task because attention has to shift to the position of the stimulus in order to classify the relevant stimulus feature. Spatial information processing occurs because the task is to classify the direction of an arrow or the spatial meaning of a word. In contrast, spatial attention and spatial information processing play little or no role in the color Stroop task, and typical alerting-congruency interactions have not been obtained with it (Schneider, 2019a; Soutschek et al., 2013; Weinbach \& Henik, 2012).

A reviewer astutely noted that a significant effect for one task and a nonsignificant effect for another task do not necessarily imply a significant difference in effects between tasks (Gelman \& Stern, 2006; Nieuwenhuis, Forstmann, \& Wagenmakers, 2011). However, the apparent difference in the alerting-congruency interaction patterns for the spatial and color Stroop tasks receives statistical support when data from the two tasks are analyzed together. I conducted an exploratory analysis involving the spatial Stroop task data from the present Experiments 3 and $4(N=120)$, and the color Stroop task data from Experiments $1-3(N=258)$ of Schneider (2019a). These experiments were selected for this analysis because they all used word stimuli for which the relevant and irrelevant stimulus features were spatially integrated. In the present Experiments 3 and 4, a spatial word (e.g., $L E F T$ ) was the relevant feature and its position was the irrelevant feature. In Experiments 1-3 of Schneider (2019a), a color word (e.g., RED) was the irrelevant feature and its physical color was the relevant feature.

I submitted the RT data from both sets of experiments to a mixed ANOVA with task (spatial Stroop or color Stroop) as a between-subjects factor, and alerting (no alert or alert) and congruency (incongruent or congruent) as within-subjects factors. The key result was a significant three-way interaction, $F(1,376)=17.10, p<.001, \eta_{\mathrm{p}}^{2}=.043$, which reflects the different alerting-congruency interaction patterns across tasks: The mean difference in congruency effects between alert and no-alert trials was $17 \mathrm{~ms}$ for the spatial Stroop task and $-1 \mathrm{~ms}$ for the color Stroop task. ${ }^{11}$ The different interaction patterns in different versions of the Stroop task represent complementary evidence supporting a role for spatial information processing in producing the interaction.

For the variants of the spatial Stroop task in the present study, the alerting-congruency interaction was larger for word stimuli than for arrow stimuli (compare the top and bottom panels of Fig. 3). Interestingly, the overall alerting effect - the difference in RTs between no-alert and alert trials - was smaller for word stimuli than for arrow stimuli. Considering that the same alerting cues were used with both types of stimuli (see Fig. 2), the quantitative difference in the alerting-congruency interaction patterns must reflect some aspect of how alertness influences stimulus processing.

Experimental and modeling work supports the idea that increased alertness makes stimulus encoding faster (e.g., Bundesen, Vangkilde, \& Habekost, 2015; Matthias et al., 2010; Petersen, Petersen, Bundesen, Vangkilde, \& Habekost, 2017; Rolke \& Hofmann, 2007; Seifried, Ulrich, Bausenhart, Rolke, \& Osman, 2010). Based on this idea, Nieuwenhuis and de Kleijn (2013) modeled the alerting-congruency interaction in the flanker task by assuming that a shorter stimulus encoding time allows stimulus classification and response selection to start sooner, when attention is relatively unfocused. However, the process of extracting spatial information from the encoded stimulus for classification depends on the type of stimulus, and there is evidence that spatial information is processed differently for spatial words and arrows (e.g., Gibson \& Kingstone, 2006; Hommel, Pratt, Colzato, \& Godijn, 2001). If the time course of spatial information processing differs for words and arrows, and if alertness affects the time when such processing commences, then it is possible that the resulting alerting-congruency interaction will vary in magnitude across stimulus types. Computational modeling could be a fruitful approach for investigating this possibility.

\footnotetext{
${ }^{11}$ Previous analyses for individual experiments already established that the alerting-congruency interaction was significant for the spatial Stroop task (see Table 2) and nonsignificant for the color Stroop task (see Schneider, 2019a).
} 


\section{Implications for previous hypotheses}

The recurrent finding of a reliable alerting-congruency interaction in the spatial Stroop task has important implications for previous hypotheses about the interaction, most of which were based on results from the arrow flanker task. One hypothesis is that increased alertness is associated with a more diffuse focus of spatial attention, resulting in more distractor processing that produces larger congruency effects on alert trials than on noalert trials in the flanker task (McConnell \& Shore, 2011; Nieuwenhuis \& de Kleijn, 2013; Weinbach \& Henik, 2012). However, the diffuse attention hypothesis has not been supported in recent tests (Schneider, 2018b; Seibold, 2018), and it is unclear how it would explain alerting-congruency interactions in the spatial Stroop task or the Simon task, where the irrelevant feature (stimulus position) is not spatially separated from the relevant feature. Weinbach and Henik (2012, p. 1538) suggested that "broadening the attentional beam could increase the [cognitive] system's sensitivity to the spatial location of the target" in the Simon task. They did not elaborate on the nature of this increased sensitivity to spatial location, but one possibility is that a wider focus of attention is more likely to encompass both the target stimulus and a central fixation sign, with the latter serving as a reference point for easier coding of stimulus position. This idea would be consistent with referential coding accounts of Simon task performance (e.g., Hommel, 1993).

As an alternative to diffuse attention, Schneider (2018b) hypothesized that increased alertness might be associated with a higher probability of spatially grouping the distractors with the target in the flanker task, thereby producing an alertingcongruency interaction. However, the spatial grouping hypothesis was not supported in recent experiments in which spatial grouping was disrupted by misaligning stimuli in the flanker task (Schneider, 2019b), and it also cannot account for alerting-congruency interactions in the spatial Stroop task or the Simon task, where spatial grouping does not occur.

An idea that seems consistent with the present data is the facilitated response activation hypothesis of Fischer, Plessow, and Kiesel (2012; see also Böckler et al., 2011). They proposed that increased alertness facilitates a direct route for response selection involving established stimulus-response associations. When stimuli are strongly associated with specific response codes (e.g., arrow stimuli and spatial responses in the arrow flanker task), heightened alertness is hypothesized to boost activation along these direct links, amplifying the contribution of distractors to response selection and producing an alerting-congruency interaction. In support of the hypothesis, Fischer et al. (2012) found that the interaction occurred reliably in a word flanker task only when the distractor words also served occasionally as target words, which allowed the formation of direct stimulus-response associations for the distractors. Beyond the word flanker task, the facilitated response activation hypothesis can explain data from the Simon and spatial Stroop tasks if alerting modulates the activation of established links between the irrelevant stimulus positions and spatial response codes.

However, it is less clear how the hypothesis can account for the data in Schneider (2019a) from color Stroop-like tasks involving distractors with preexisting spatial associations (e.g., making a left or right key-press response to classify the color of a rectangle flanked above and below by the word LEFT or RIGHT). According to the facilitated response activation hypothesis, increased alertness should boost activation from a distractor (e.g., LEFT) to its associated spatial code ("left") that overlaps with a response code (left key press), thereby increasing congruency effects. I obtained reliable congruency effects in three experiments involving color Strooplike tasks (Experiments 6-8 in Schneider, 2019a), indicating that the distractors influenced response selection, but the congruency effects were not larger on alert trials than on no-alert trials.

\section{Considering dimensional overlap}

A reviewer suggested that the nature of the dimensional overlap between stimuli and responses might play a role in determining whether the alerting-congruency interaction will be obtained for a given task. Kornblum and colleagues proposed a taxonomy of stimulus-response ensembles for classifying the overlap of relevant and irrelevant stimulus dimensions and response sets (e.g., Kornblum \& Lee, 1995; Kornblum, Stevens, Whipple, \& Requin, 1999). In their taxonomy, all variants of the spatial Stroop task in the present study involve Type 8 ensembles because the relevant stimulus dimension (e.g., arrow direction), irrelevant stimulus dimension (e.g., arrow position), and response set (e.g., pressing a left or right key) all overlap with each other. The arrow versions of the flanker and global/local tasks also involve Type 8 ensembles. Alerting-congruency interactions have been consistently found with these tasks (e.g., Callejas et al., 2005; Fan et al., 2002; J. W. MacLeod et al., 2010; McConnell \& Shore, 2011; Redick \& Engle, 2006; Schneider, 2018a, 2018b, 2019b; Weinbach \& Henik, 2011, 2012, 2014).

In contrast, the color Stroop task with left-right manual responses involves Type 4 ensembles because there is overlap between the relevant and irrelevant stimulus dimensions (stimulus color and word meaning, respectively), but neither overlaps with the response set. Alerting-congruency interactions have not been found with this task (Schneider, 2019a; Soutschek et al., 2013; Weinbach \& Henik, 2012). The color Stroop task can be modified to involve Type 8 ensembles if vocal responses (color names) are used instead of manual responses. If the alerting-congruency interaction routinely occurs with Type 8 ensembles, then it might emerge in the color Stroop task with vocal responses. A caveat to this prediction is 
that previous research has revealed that vocal responding produces larger congruency effects than does manual responding, as well as a different pattern of performance across a range of stimulus types (e.g., Kinoshita, De Wit, \& Norris, 2017; Sharma \& McKenna, 1998). Consequently, a change in response modality would likely alter Stroop task processing (in addition to changing the type of stimulus-response ensemble), and the implications for finding an alerting-congruency interaction are unknown.

There is mixed evidence concerning alerting-congruency interactions for Type 4 ensembles in non-Stroop tasks. For example, many flanker tasks with nonarrow stimuli (e.g., letters) and left-right manual responses involve Type 4 ensembles. Reliable alerting-congruency interactions have been obtained in some experiments with nonarrow flanker tasks, but not in others (for a summary, see Schneider, 2019a). One potentially important factor might be whether the nonarrow stimuli have directional associations that can be mapped onto the spatial dimension used in responding (Kahan \& Zhang, 2019).

Even if the alerting-congruency interaction turns out to be prevalent with Type 8 ensembles (and present under specific circumstances with Type 4 ensembles), there is already evidence that it is not restricted to those ensembles. The Simon task involves Type 3 ensembles because there is overlap between the irrelevant stimulus dimension (stimulus position) and the response set (pressing a left or right key), but neither overlaps with the relevant stimulus dimension (e.g., stimulus shape). Alerting-congruency interactions have been consistently found with the Simon task (Böckler et al., 2011; Fischer et al., 2010; R. M. Klein \& Ivanoff, 2011; Soutschek et al., 2013). However, other tasks involving Type 3 ensembles have not yielded the interaction. For example, the Strooplike tasks in Experiments 7 and 8 of Schneider (2019a) involved Type 3 ensembles because there was overlap between the irrelevant stimulus dimension (spatial word or arrow distractor) and the response set (pressing a left or right key), but neither overlapped with the relevant stimulus dimension (target stimulus color). An alerting-congruency interaction was not found in either experiment, despite high statistical power.

The preceding taxonomic analysis indicates that the alerting-congruency interaction is not limited to one specific type of stimulus-response ensemble. However, the available evidence suggests that it is difficult to predict whether the interaction will be obtained with a given ensemble, with the possible exception of Type 8 ensembles, which involve complete overlap between the relevant and irrelevant stimulus dimensions and the response set. Future research focusing on the circumstances under which the interaction is present or absent for an ensemble might lead to new or refined hypotheses of the alerting-congruency interaction. This is important because the collective results from my laboratory's experiments involving the arrow flanker task (10 experiments:
Schneider, 2018a, 2018b, 2019b), color Stroop task (eight experiments: Schneider, 2019a), and spatial Stroop task (four experiments: present study) cannot be fully accommodated by any existing hypothesis of the interaction. My previous findings led me to suggest that spatial attention and spatial information processing are important sources of the interaction (Schneider, 2019a), and this suggestion is reinforced by the results from the spatial Stroop task in the present study. Future empirical and theoretical work that focuses on spatial information processing, especially in the context of different tasks and with different types of stimulus-response ensembles, might contribute to more progress in understanding how alertness is related to cognitive control.

Author note I thank Lara David, Nimisha Prasad, Analicia Rios, Taylor Springer, and Julia Terlep for assistance with data collection.

Open practices statement The data and materials for all experiments are available upon request from the author. All experiments were preregistered (see footnote 5).

\section{References}

Böckler, A., Alpay, G., \& Stürmer, B. (2011). Accessory stimuli affect the emergence of conflict, not conflict control: A Simon-task ERP study. Experimental Psychology, 58, 102-109.

Bundesen, C., Vangkilde, S., \& Habekost, T. (2015). Components of visual bias: A multiplicative hypothesis. Annals of the New York Academy of Sciences, 1339, 116-124.

Callejas, A., Lupiáñez, J., Funes, M. J., \& Tudela, P. (2005). Modulations among the alerting, orienting, and executive control networks. Experimental Brain Research, 167, 27-37.

Callejas, A., Lupiáñez, J., \& Tudela, P. (2004). The three attentional networks: On their independence and interactions. Brain and Cognition, 54, 225-227.

Clark, H. H., \& Brownell, H. H. (1975). Judging up and down. Journal of Experimental Psychology: Human Perception and Performance, 1 , 339-352.

Craft, J. L., \& Simon, J. R. (1970). Processing symbolic information from a visual display: Interference from an irrelevant directional cue. Journal of Experimental Psychology, 83, 415-420.

Eriksen, B. A., \& Eriksen, C. W. (1974). Effects of noise letters upon the identification of a target letter in a nonsearch task. Perception \& Psychophysics, 16, 143-149.

Fan, J., McCandliss, B. D., Sommer, T., Raz, A., \& Posner, M. I. (2002). Testing the efficiency and independence of attentional networks. Journal of Cognitive Neuroscience, 14, 340-347.

Faul, F., Erdfelder, E., Lang, A.-G., \& Buchner, A. (2007). G*Power 3: A flexible statistical power analysis program for the social, behavioral, and biomedical sciences. Behavior Research Methods, 39, 175-191.

Fischer, R., Plessow, F., \& Kiesel, A. (2010). Auditory warning signals affect mechanisms of response selection: Evidence from a Simon task. Experimental Psychology, 57, 89-97.

Fischer, R., Plessow, F., \& Kiesel, A. (2012). The effects of alerting signals in action control: Activation of S-R associations or inhibition of executive control processes? Psychological Research, 76, 317-328

Gelman, A., \& Stern, H. (2006). The difference between "significant" and "not significant" is not itself statistically significant. The American Statistician, 60, 328-331. 
Gibson, B. S., \& Kingstone, A. (2006). Visual attention and the semantics of space: Beyond central and peripheral cues. Psychological Science, 17, 622-627.

Hommel, B. (1993). The role of attention for the Simon effect. Psychological Research, 55, 208-222.

Hommel, B., Pratt, J., Colzato, L., \& Godijn, R. (2001). Symbolic control of visual attention. Psychological Science, 12, 360-365.

Kahan, T. A., \& Zhang, H. (2019). Ready to be distracted: Further evidence that the alerting-congruency interaction requires stimulusresponse directional associations. Visual Cognition, 27(9/10), 760 767. doi:https://doi.org/10.1080/13506285.2019.1680586

Kinoshita, S., De Wit, B., \& Norris, D. (2017). The magic of words reconsidered: Investigating the automaticity of reading colorneutral words in the Stroop task. Journal of Experimental Psychology: Learning, Memory, and Cognition, 43, 369-384.

Klein, R. A., Vianello, M, Hasselman, F., Adams, B. G., Adams, R. B., Alper, S., ... Nosek, B. A. (2018). Many Labs 2: Investigating variation in replicability across samples and settings. Advances in Methods and Practices in Psychological Science, 1, 443-490. doi: https://doi.org/10.1177/2515245918810225

Klein, R. M., \& Ivanoff, J. (2011). The components of visual attention and the ubiquitous Simon effect. Acta Psychologica, 136, 225-234.

Kornblum, S., \& Lee, J.-W. (1995). Stimulus-response compatibility with relevant and irrelevant stimulus dimensions that do and do not overlap with the response. Journal of Experimental Psychology: Human Perception and Performance, 21, 855-875.

Kornblum, S., Stevens, G. T., Whipple, A., \& Requin, J. (1999). The effects of irrelevant stimuli: 1 . The time course of stimulusstimulus and stimulus-response consistency effects with Strooplike stimuli, Simon-like tasks, and their factorial combinations. Journal of Experimental Psychology: Human Perception and Performance, 25, 688-714.

Logan, G. D., \& Zbrodoff, N. J. (1979). When it helps to be misled: Facilitative effects of increasing the frequency of conflicting stimuli in a Stroop-like task. Memory \& Cognition, 7, 166-174.

Lu, C.-H., \& Proctor, R. W. (1995). The influence of irrelevant location information on performance: A review of the Simon and spatial Stroop effects. Psychonomic Bulletin \& Review, 2, 174-207.

Lukas, S., Philipp, A. M., \& Koch, I. (2010). The role of preparation and cue-modality in crossmodal task switching. Acta Psychologica, 134, 318-322.

MacLeod, C. M. (1991). Half a century of research on the Stroop effect: An integrative review. Psychological Bulletin, 109, 163-203.

MacLeod, J. W., Lawrence, M. A., McConnell, M. M., Eskes, G. A., Klein, R. M., \& Shore, D. I. (2010). Appraising the ANT: Psychometric and theoretical considerations of the Attention Network Test. Neuropsychology, 24, 637-651.

Masson, M. E. J., \& Loftus, G. R. (2003). Using confidence intervals for graphically based data interpretation. Canadian Journal of Experimental Psychology, 57, 203-220.

Matthias, E., Bublak, P., Müller, H. J., Schneider, W. X., Krummenacher, J., \& Finke, K. (2010). The influence of alertness on spatial and nonspatial components of visual attention. Journal of Experimental Psychology: Human Perception and Performance, 36, 38-56.

McConnell, M. M., \& Shore, D. I. (2011). Mixing measures: Testing an assumption of the attention network test. Attention, Perception, \& Psychophysics, 73, 1096-1107.

Navon, D. (1977). Forest before trees: The precedence of global features in visual perception. Cognitive Psychology, 9, 353-383.

Nieuwenhuis, S., \& de Kleijn, R. (2013). The impact of alertness on cognitive control. Journal of Experimental Psychology: Human Perception and Performance, 39, 1797-1801.
Nieuwenhuis, S., Forstmann, B. U., \& Wagenmakers, E.-J. (2011). Erroneous analyses of interactions in neuroscience: A problem of significance. Nature Neuroscience, 14, 1105-1107.

Open Science Collaboration. (2015). Estimating the reproducibility of psychological science. Science, 349(6251), aac4716.

Palef, S. R., \& Olson, D. R. (1975). Spatial and verbal rivalry in a Strooplike task. Canadian Journal of Psychology, 29, 201-209.

Palmer, S. E. (1999). Vision science: Photons to phenomenology. Cambridge, MA: MIT Press.

Petersen, A., Petersen, A. H., Bundesen, C., Vangkilde, S., \& Habekost, T. (2017). The effect of phasic auditory alerting on visual perception. Cognition, 165, 73-81.

Posner, M. I. (2008). Measuring alertness. Annals of the New York Academy of Sciences, 1129, 193-199.

Posner, M. I., \& Boies, S. J. (1971). Components of attention. Psychological Review, 78, 391-408.

Posner, M. I., \& Petersen, S. E. (1990). The attention system of the human brain. Annual Review of Neuroscience, 13, 25-42.

Quinlan, P. T., \& Hill, N. I. (1999). Sequential effects in rudimentary auditory and visual tasks. Perception \& Psychophysics, 61, 375384.

Redick, T. S., \& Engle, R. W. (2006). Working memory capacity and attention network test performance. Applied Cognitive Psychology, $20,713-721$

Rolke, B., \& Hofmann, P. (2007). Temporal uncertainty degrades perceptual processing. Psychonomic Bulletin \& Review, 14, 522-526.

Rouder, J. N., Speckman, P. L., Sun, D., Morey, R. D., \& Iverson, G. (2009). Bayesian $t$ tests for accepting and rejecting the null hypothesis. Psychonomic Bulletin \& Review, 16, 225-237.

Schneider, D. W. (2018a). Alertness and cognitive control: Testing the early onset hypothesis. Journal of Experimental Psychology: Human Perception and Performance, 44, 756-766.

Schneider, D. W. (2018b). Alertness and cognitive control: Toward a spatial grouping hypothesis. Attention, Perception, \& Psychophysics, 80, 913-928.

Schneider, D. W. (2019a). Alertness and cognitive control: Is there a spatial attention constraint? Attention, Perception, \& Psychophysics, 81, 119-136.

Schneider, D. W. (2019b). Alertness and cognitive control: Testing the spatial grouping hypothesis. Attention, Perception, \& Psychophysics, 81, 1913-1925.

Schönbrodt, F. D., \& Wagenmakers, E.-J. (2018). Bayes factor design analysis: Planning for compelling evidence. Psychonomic Bulletin \& Review, 25, 128-142.

Seibold, V. C. (2018). Do alerting signals increase the size of the attentional focus? Attention, Perception, \& Psychophysics, 80, 402-425.

Seifried, T., Ulrich, R., Bausenhart, K. M., Rolke, B., \& Osman, A. (2010). Temporal preparation decreases perceptual latency: Evidence from a clock paradigm. Quarterly Journal of Experimental Psychology, 63, 2432-2451.

Sharma, D., \& McKenna, F. P. (1998). Differential components of the manual and vocal Stroop tasks. Memory \& Cognition, 26, 10331040.

Siegler, R. S., \& Lortie-Forgues, H. (2017). Hard lessons: Why rational number arithmetic is so difficult for so many people. Current Directions in Psychological Science, 26, 346-351.

Simon, J. R., \& Small, A. M. (1969). Processing auditory information: Interference from an irrelevant cue. Journal of Applied Psychology, 53, 433-435.

Soutschek, A., Müller, H. J., \& Schubert, T. (2013). Conflict-specific effects of accessory stimuli on cognitive control in the Stroop task and the Simon task. Experimental Psychology, 60, 140-147.

Stroop, J. R. (1935). Studies of interference in serial verbal reactions. Journal of Experimental Psychology, 18, 643-662. 
Weinbach, N., \& Henik, A. (2011). Phasic alertness can modulate executive control by enhancing global processing of visual stimuli. Cognition, 121, 454-458.

Weinbach, N., \& Henik, A. (2012). The relationship between alertness and executive control. Journal of Experimental Psychology: Human Perception and Performance, 38, 1530-1540.

Weinbach, N., \& Henik, A. (2014). Alerting enhances attentional bias for salient stimuli: Evidence from a global/local processing task. Cognition, 133, 414-419.
Zhong, Q., Xiong, A., Vu, K.-P. L., \& Proctor, R. W. (2018). Vertically arrayed stimuli and responses: Transfer of incompatible spatial mapping to Simon task occurs regardless of response-device orientation. Experimental Brain Research, 236, 175-185.

Publisher's note Springer Nature remains neutral with regard to jurisdictional claims in published maps and institutional affiliations. 